\title{
Adaptive pumping for spectral control of random lasers
}

\author{
Nicolas Bachelard ${ }^{1}$, Sylvain Gigan ${ }^{1}$, Xavier Noblin $^{2}$ and Patrick Sebbah ${ }^{1 \star}$
}

\begin{abstract}
A laser is not necessarily a sophisticated device: pumping an amplifying medium randomly filled with scatterers makes a perfectly viable 'random laser'. The absence of mirrors greatly simplifies laser design, but control over the emission wavelength and directionality is lost, seriously hindering prospects $^{1-4}$ for this otherwise simple laser. Recently, we proposed an approach to tame random lasers ${ }^{5}$, inspired by coherent light control in complex media ${ }^{6}$. Here, we implement this method in an optofluidic random laser ${ }^{7}$ where modes are spatially extended and overlap, making individual mode selection impossible, a priori. We show experimentally that control over laser emission can be regained even in this extreme case. By actively shaping the optical pump within the random laser, single-mode operation at any selected wavelength is achieved with spectral selectivity down to $0.06 \mathrm{~nm}$ and more than $10 \mathrm{~dB}$ side-lobe rejection. This method paves the way towards versatile tunable and controlled random lasers as well as the taming of other laser sources.
\end{abstract}

In conventional lasers, the optical cavity determines the main characteristics of the laser emission: its geometry fixes the emission wavelengths and the lasing modes, as well as the direction of the laser emission. The active medium itself essentially provides the optical gain when pumped by an external source. There are, however, situations where laser emission is not fully controlled by the optical cavity. This is the case in mirrorless lasers, such as random lasers, where the optical feedback is provided solely by multiple scattering within the gain medium ${ }^{1-4}$. Coherent laser emission has been reported in various active random media, raising strong interest for this class of lasing sources, which are easy to fabricate and have demonstrated unique properties and promising applications ${ }^{8}$. The absence of a well-defined cavity, however, results in an unpredictable random emission spectrum and multi-directionality. If the scattering is not strong enough to tightly confine the modes ${ }^{9-11}$, they overlap and the situation gets even worse: strong mode competition and temporal chaotic behaviour are expected $^{12-15}$, seriously limiting practical interest in random lasers.

In this Letter, we achieve experimental control of the random laser spectral emission by optimization of the optical pump profile. The first proposal for laser optimization based on non-uniform pumping can be traced back to the seminal paper by Kogelnik and Shank ${ }^{16}$, where it was suggested in the conclusion that, instead of applying a spatial modulation to the index of refraction, the pump intensity could be spatially modulated to vary the gain periodically and realize a distributed feedback (DFB) laser. This idea was next applied by the same authors to tune the emission wavelength of a DFB laser ${ }^{17}$. Other than this example, the spatial modulation of the optical pump intensity has not been widely adopted. Optimization of electrical pumping is tested, for example, in high-speed single-mode
DFB semiconductor lasers ${ }^{18}$. But, in these systems, the structured electrodes are fixed, precluding broad tunability. Recently, emission control was demonstrated in a particular micro-random laser where modes could be selected independently: the random laser was pumped uniformly below threshold and the pump was shaped into a directive wedged channel to externally feed the random laser in a particular direction ${ }^{19}$. In contrast, the method we propose is generic for random lasers, and moreover is not limited to random lasers. We optimally shape the pump within the random medium to gain in complexity and achieve control at all lasing wavelengths, even in the weakly scattering regime where modes are spatially extended and selection by local or directional pumping as in ref. 19 is precluded. We successfully tested this method numerically ${ }^{5}$. However, the model used did not include mode competition, gain saturation and spectral fluctuations. Here, we experimentally show that active control of the spatial pump profile using a spatial light modulator is an effective method in practice that survives shot-toshot fluctuations and strong nonlinear behaviour. We are able to bring a random laser from multimode to single-mode operation at any of our selected emission wavelengths. Surprisingly, laser emission control via pump shaping is easily achieved despite spatial overlap and non-orthogonality of the modes. Our method extends wavefront shaping techniques for imaging through opaque media to the control of open, disordered, nonlinear active media. It shows that complexity is not necessarily an obstacle, but may help in meeting the optimization criterion ${ }^{20}$. The experiment reveals how mode competition is precisely tailored by the optimized pump profile to achieve mode selection.

We consider the optically pumped one-dimensional (1D) random laser described in Fig. 1, inspired by an optofluidic random laser we recently introduced ${ }^{7}$ for easy integration of lasers into complex optofluidic structures ${ }^{21}$. The pump laser is reflected off a computer-driven spatial light modulator (SLM) to modulate its spatial intensity profile and is focused into a narrow strip line to pump the dye. The optical set-up is described in more detail in the Methods. Figure 2a,b compares the emission spectra integrated over 50 shots, $I(\lambda)$, versus wavelength $\lambda$, just below threshold and above threshold, respectively. Below threshold, amplified spontaneous emission (ASE) results in a broad spectral peak. When the pump exceeds the threshold, sharp peaks emerge in the spectrum. Their measured spectral linewidth is $0.03 \mathrm{~nm}$ (fullwidth at half-maximum) and is not limited by the 20 pm-resolution of the imaging spectrometer (Methods). The number of peaks in the frequency range $560-565 \mathrm{~nm}$ is $35 \pm 3$ for ten sample realizations. This is consistent with the spectral density of lasing modes found numerically (Supplementary Methods). Single-mode operation is, however, extremely difficult to achieve when the random laser is uniformly pumped, as mode thresholds are very close. However,

\footnotetext{
${ }^{1}$ Institut Langevin, ESPCI ParisTech, CNRS UMR7587, 1 rue Jussieu, 75238 Paris cedex 05, France, ${ }^{2}$ Laboratoire de Physique de la Matière Condensée, Université Nice-Sophia Antipolis, CNRS UMR 7336, Parc Valrose, 06108 Nice cedex 02, France. *e-mail: patrick.sebbah@espci.fr
} 
a

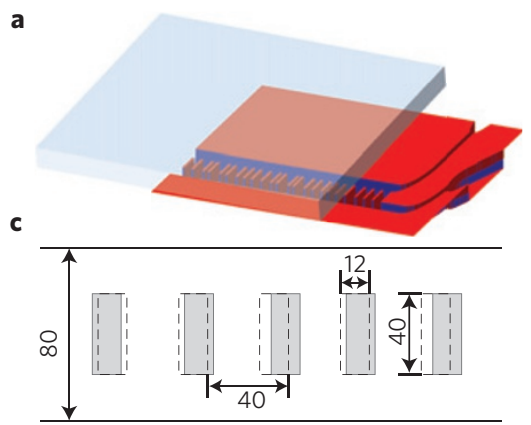

b

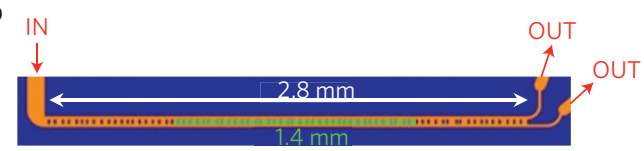

d

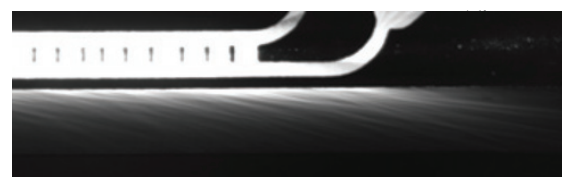

Figure 1 | Optofluidic random laser. a, 3D partial view of the mask. b, Complete top view of the mask. c, The microchannel is structured into a 1D-random distribution of $12 \mu \mathrm{m}$-thick rectangular pillars separated on average by $28 \mu \mathrm{m}$ gaps. Geometrical dimensions are given in $\mu \mathrm{m}$. A dye solution (Rhodamine $6 \mathrm{G}$ in ethanol) flows through the structured PDMS microchannel, which is plasma-bonded on a glass slide partially represented in a. A pressure differential at the two outlets forces dye flow between the scattering pillars and prevents dye bleaching. The structure is pumped by a Q-switched Nd:YAG laser at $532 \mathrm{~nm}$ shaped into a $1.4 \mathrm{~mm}$-long stripe line (green line in b). d, Imaging of the in-plane scattered laser emission. Dye (Nile blue) solution circulates in an $80 \mu \mathrm{m}$-wide auxiliary microchannel parallel to and at a distance of $10 \mu \mathrm{m}$ from the structured channel and fluoresces at the laser emission wavelength.

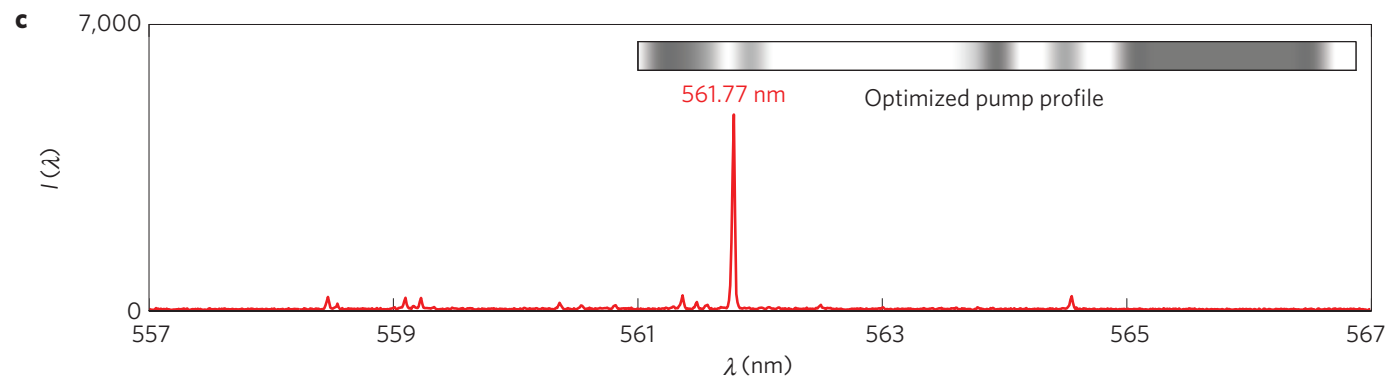

b
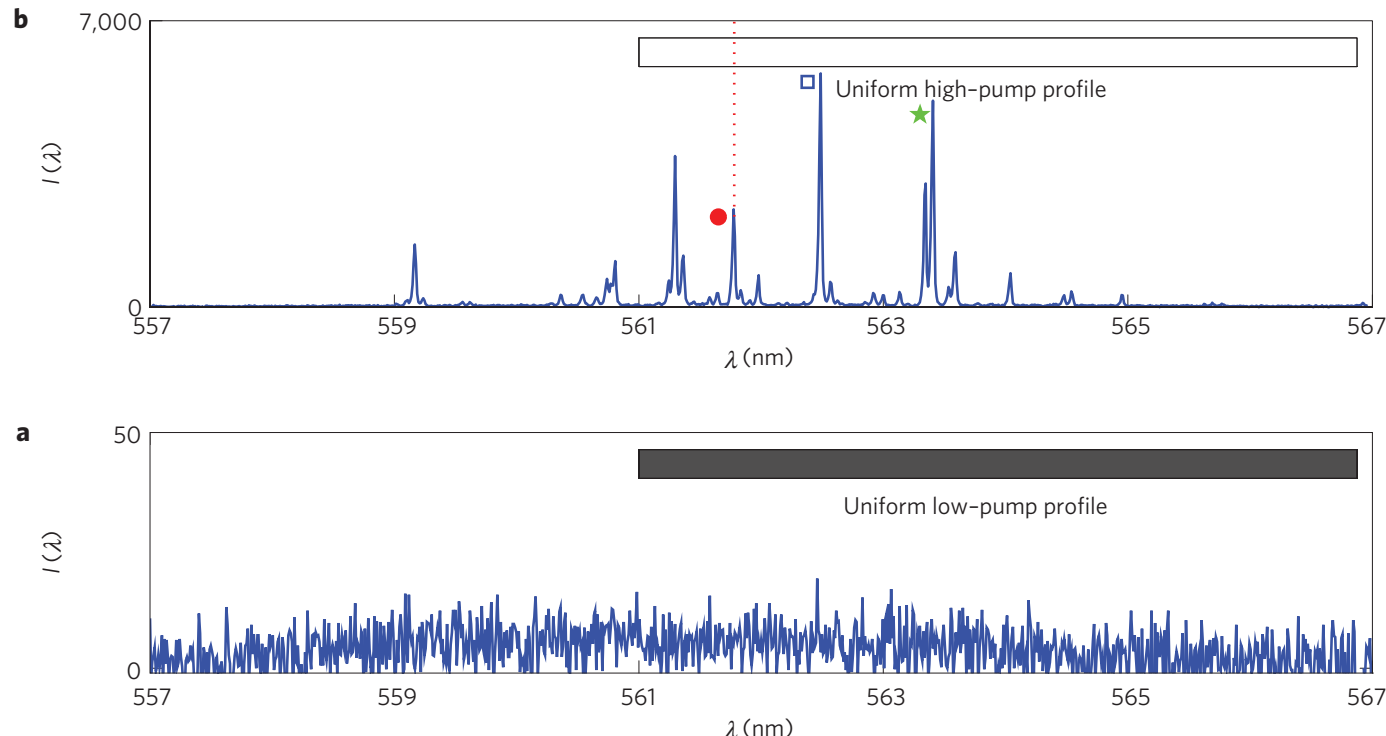

Figure $\mathbf{2}$ | From multimode to single-mode operation. a, Fluorescence spectrum for uniform pumping below threshold. b, Laser emission spectrum for uniform pumping above threshold. Symbols correspond to the modes shown in Fig. 5. c, Emission spectrum after the optimization process to select laser emission at $\lambda_{0}=561.77 \mathrm{~nm}$. Single-mode operation is achieved after 228 iterations. The corresponding nonuniform pump profile as displayed on the spatial light modulator (SLM) is shown in the inset. The grey scale ranges from 0 (black) to 255 (white).

we claim that the sensitivity of the emission spectrum to global and local variations of the optical pump profile ${ }^{22,23}$ should enable single-mode laser operation at a selected wavelength, $\lambda_{0}$. To do so, we need to find the pump profile, if it exists, that maximizes the ratio $R\left(\lambda_{0}\right)=I\left(\lambda_{0}\right) / I\left(\lambda_{1}\right)$, where $\lambda_{1}$ is the emission wavelength of the lasing mode with highest intensity, apart from the mode at $\lambda_{0}$. A $R\left(\lambda_{0}\right)$ much larger than unity signifies that the mode at $\lambda_{0}$ has been selected and that single-mode operation at this wavelength is possible.

At this point, it is important to discuss the nature of scattering within our random laser. The small index contrast between the polydimethylsiloxane (PDMS) pillars and the dye solution, $\Delta n=1.42-1.36=0.06$, results in weak scattering. This is confirmed numerically by computing the localization length 


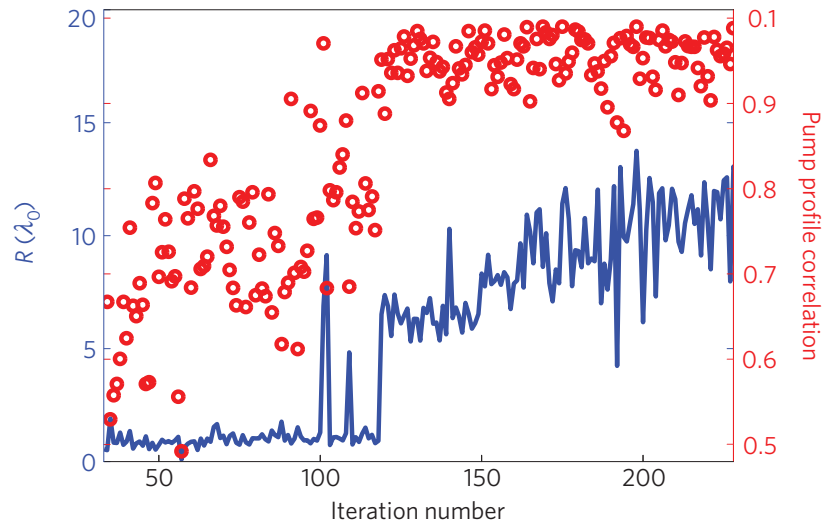

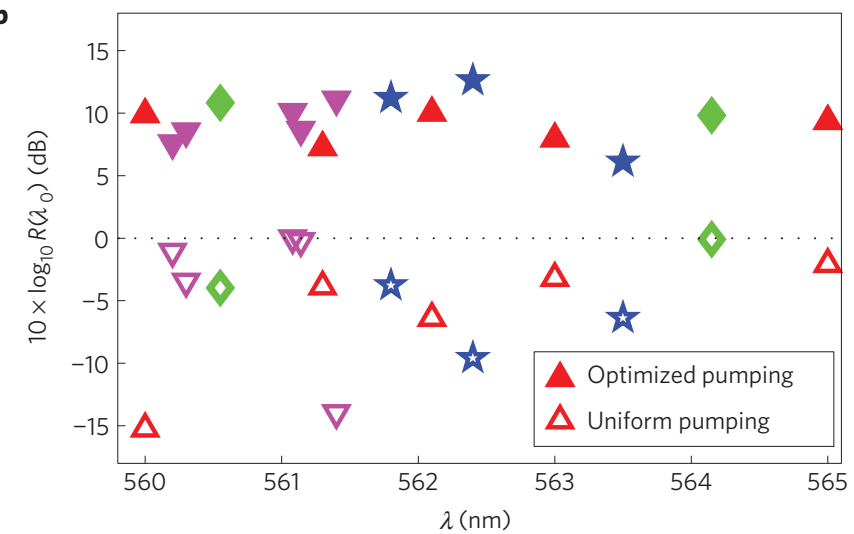

Figure 3 | Convergence and efficiency of the optimization process. a, Evolution versus iteration number of the inverse cost function $R\left(\lambda_{0}\right)(b l u e$ line) and the normalized spatial correlation between iterations $i$ and $i+1$ of the spatial profile of the pump (open circles), for the optimization of the mode at $\lambda_{0}=561.77 \mathrm{~nm}$. Despite large experimental fluctuations and wide exploration of the solution space, convergence is eventually reached when the modulated pump profile stabilizes. $\mathbf{b}$, Inverse cost function $R\left(\lambda_{0}\right)$ versus wavelength before (open symbols: $\left.R\left(\lambda_{0}\right)<1\right)$ and after (full symbols: $\left.R\left(\lambda_{0}\right) \gg 1\right)$ optimization of 15 lasing modes. The logarithmic representation gives $R\left(\lambda_{0}\right)$ in $\mathrm{dB}$. The different symbols correspond to different sets of experiments (Methods).

$\xi=-(\mathrm{d}\langle\ln T\rangle / \mathrm{d} L)^{-1}$ from the dependence of the transmission $T$ of an ensemble of 100 random configurations with sample length $L$ (ref. 22). We find $\xi=22 \mathrm{~mm}$ at $560 \mathrm{~nm}$, much longer than the sample size $(2.8 \mathrm{~mm})$. In this regime, the modes extend over the whole system and therefore strongly overlap with each other. Hence, it is impossible a priori to predict the correct pump profile required to select the targeted mode at $\lambda_{0}$ at the expense of all others. Although linear methods fail, we have shown numerically that an iterative algorithm can provide an optimized solution ${ }^{5}$. However, the transfer matrix model used was valid only below threshold; it did not include gain saturation, nonlinear mode competition and laser instabilities, which are expected to be all the more significant for random lasers operating in the weakly scattering regime $e^{10,11}$. In particular, shot-to-shot fluctuations and noise put experimental demonstration at a disadvantage. Here, we chose the derivativefree simplex algorithm, which is less sensitive to experimental noise than, for example, gradient-based algorithms (used in ref. 5) to minimize the cost function $1 / R\left(\lambda_{0}\right)$. At each iteration, a new pump profile is computed, displayed on the SLM and applied to the pump stripe; the spectrum averaged over 50 shots is acquired and $R\left(\lambda_{0}\right)$ is computed. After 228 iterations, the algorithm has converged to an optimized pump profile. The corresponding spectrum is shown in Fig. 2c, when the mode at $\lambda_{0}=561.77 \mathrm{~nm}$ is targeted. For demonstration, we chose a lasing mode that is not the first to lase under uniform pumping, that is, $R\left(\lambda_{0}\right)=0.4$ initially. We obtain $R\left(\lambda_{0}\right)=13.1$ after optimization, which corresponds to a sideband rejection of $11.4 \mathrm{~dB}$. The complete optimization cycle starting from uniform pumping and a multimode emission spectrum is presented in a movie (Supplementary Methods), which shows the convergence of the algorithm to a stable pump profile and a singlemode spectrum. Figure 3 a shows the convergence of $R\left(\lambda_{0}\right)$, together with the converging spatial correlation of pump profile versus iteration number. We also check that no mode-hoping between laser lines occurs during the optimization process so that the mode selected is indeed the mode initially targeted (Supplementary Methods). Actually, it is possible by this method to optimize almost any of the lasing modes present in the multimode spectrum measured with uniform pumping. The convergence criterion $R\left(\lambda_{0}\right)$ is reported in Fig. $3 b$ before and after optimization for 15 lasing modes. Optimization is efficient even away from the centre of the gain curve and even for weak lasing modes with initial $R\left(\lambda_{0}\right)$ as low as $3 \%$. Most remarkable, we succeeded in independently selecting two lasing modes separated only by $0.06 \mathrm{~nm}$, as shown in
Fig. 4. The optimization process clearly leads to two distinct pump profiles, associated with two different modes at $\lambda_{0}=561.08 \mathrm{~nm}$ and $\lambda_{0}=561.14 \mathrm{~nm}$. It is worth noting that mode selection is demonstrated here in the most extreme case of weak scattering: by extension, spectral control will be facilitated in any random laser with higher scattering, where lasing thresholds are lower, the range of single-mode operability larger and spatial discrimination easier ${ }^{5}$.

Gain nonlinearities such as mode competition and other holeburning effects play a crucial role in multimode random lasers ${ }^{14}$. This is illustrated in Fig. 5, where the mode intensity versus pump fluence is shown for the three modes indicated by symbols in Fig. 2b. Under uniform pumping (Fig. 5a), mode saturation is observed, which critically limits output intensity: modes interact to modify their spatial profile to share the gain available within the random cavity. In contrast, the non-uniform pump profile (Fig. 5b) is optimized in the sense that it breaks the parity between competing modes in favour of the targeted one. We observe that: lasing thresholds are significantly increased relative to the targeted mode (mode selection); the slope efficiency (linear fit in Fig. 5b), which measures the conversion rate of pump energy into lasing, is considerably reduced for modes other than the targeted one; emission at selected wavelength $\lambda_{0}$ is no longer saturated. These observations provide a clue to understand the nonlinear mechanism involved in the optimization process. Non-uniform pumping is actually a highly complex nonlinear process: the random active medium itself is modified at each iteration by the new pump profile. As a result, the laser output is not bounded by the parameters of the passive random system and modes gain freedom to better adjust and meet the convergence criterion. We believe that, when optimized, the pump shaping most probably redistributes the gain to tailor mode competition in an asymmetrical way: saturation is enhanced for competitive modes whereas it is strongly reduced for the targeted mode. This mechanism is fundamentally different from that of spatial or directional selective pumping ${ }^{19}$, which is precluded in the regime of strong modal overlap we consider here. The subtle mechanism revealed here calls for further theoretical investigations.

The method we have demonstrated is, in principle, scalable down to micro-lasers, as the SLM modulation can be imaged with a $\mu \mathrm{m}$-resolution. The extension to $2 \mathrm{D}$ random lasers is also possible despite the increased modal density, provided the system is small or the index contrast high enough. Once single-mode operation is achieved, the sensitivity of the medium to pump variation should allow fine tuning of the emission wavelength, turning the 

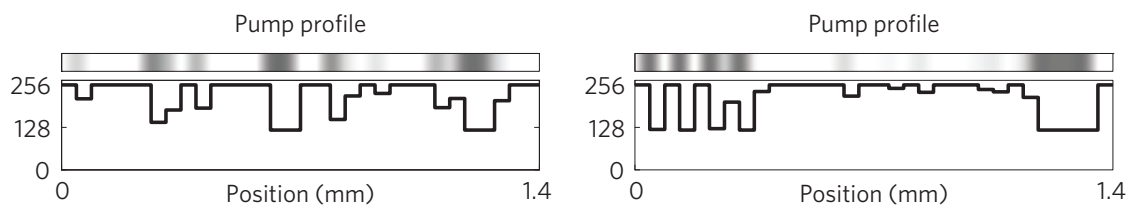

a

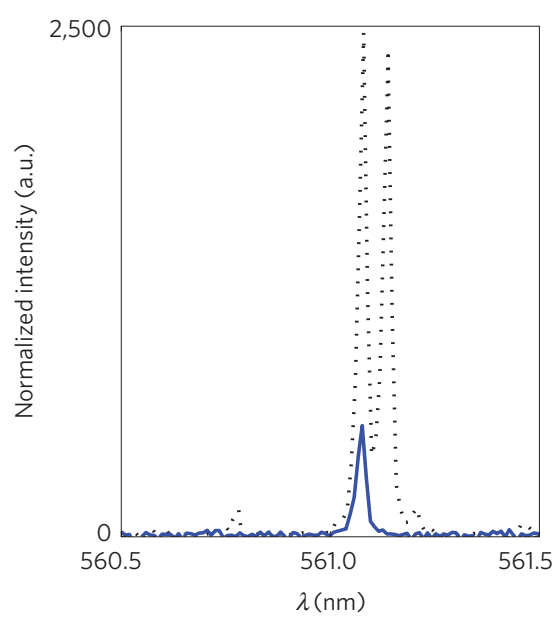

b

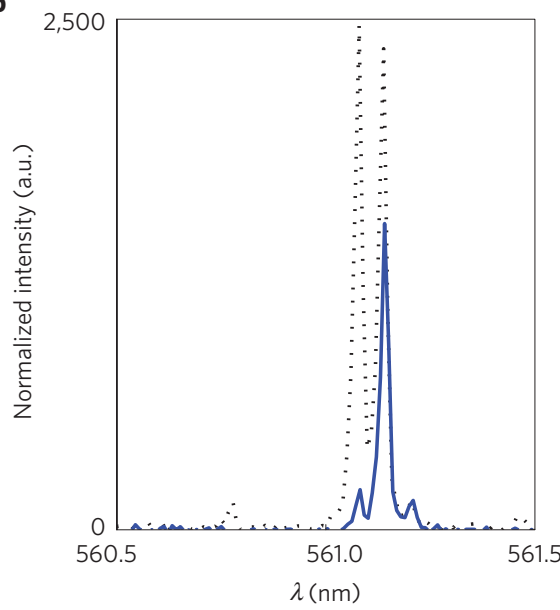

Figure 4 | Spectral selectivity. Optimizations at $\lambda_{0}=561.08 \mathrm{~nm}$ (a) and $\lambda_{0}=561.14 \mathrm{~nm}$ (b). The corresponding optimized pump profile is plotted versus position and the pattern displayed on the spatial light modulator is shown on a grey scale. Although the two modes are separated only by $0.06 \mathrm{~nm}$, the algorithm converges to two distinct spatial pump profiles, associated with two different lasing modes.

a

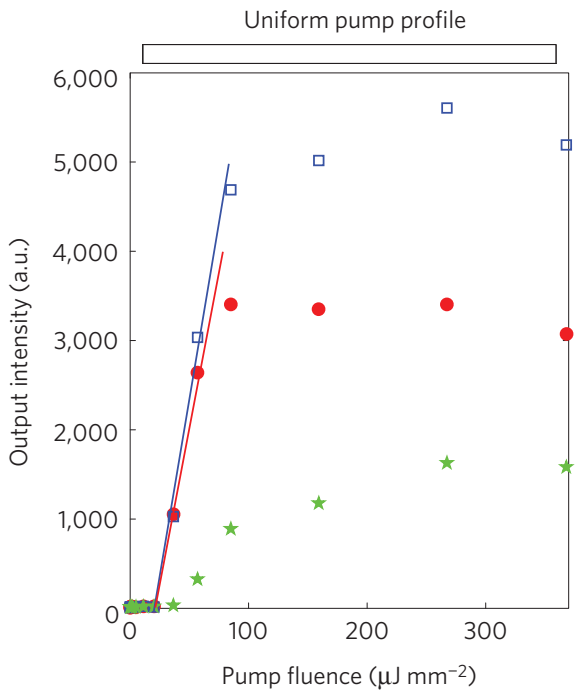

b

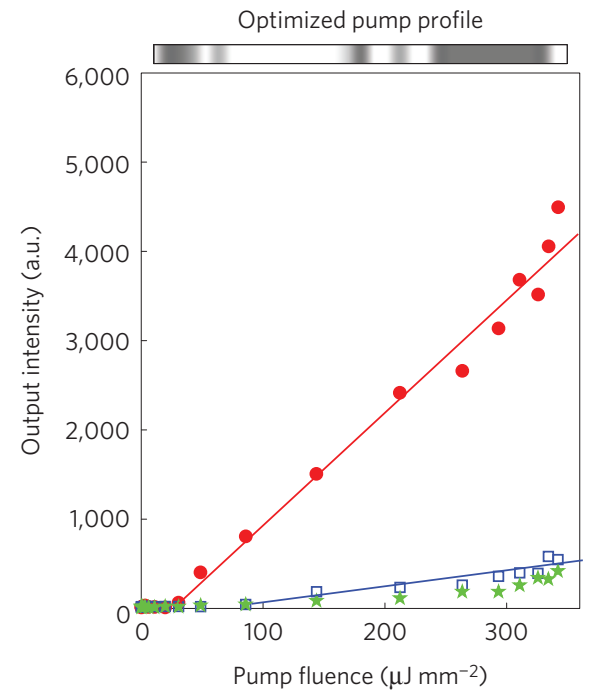

Figure 5 | Intensity versus pump fluence. Uniform pumping (a) and optimized pumping (b) for the optimization routine carried out at $\lambda_{0}=561.77 \mathrm{~nm}$. The pump profile as displayed on the spatial light modulator is shown above each plot. For the targeted mode at $\lambda_{0}=561.77 \mathrm{~nm}$ (full red circles), the threshold increased slightly from 21 to $28 \mu \mathrm{J} \mathrm{mm}{ }^{-2}$. For the mode which lases first under uniform pumping at $\lambda_{1}=562.50 \mathrm{~nm}$ (blue squares), the threshold doubled from 21 to $44 \mu \mathrm{J} \mathrm{mm}{ }^{-2}$. Green stars indicated another mode at $\lambda_{2}=563.4 \mathrm{~nm}$. Lines are a linear fit to the stimulated regimes. In $\mathbf{b}$, the $x$-axis shows the actual fluence impinging on the sample (which is different from the fluence of the incident pump beam when pumping is not uniform).

random laser into a true continuously tunable laser. Other emission properties such as directivity ${ }^{24}$, brightness or pulse duration could be controlled in a similar way to that we have demonstrated here for the control of the emission wavelength. What makes random laser control unique is that, thanks to its many degrees of freedom, the same system can yield different lasing characteristics (for example, different output wavelengths or emission patterns) controlled by the pump. This is difficult, if not impossible, to achieve with conventional lasers made with simple cavities. Beyond the control of random lasers, other classes of lasers may benefit from our method. High-power semiconductor lasers, for instance, suffer from a loss of control at high pumping rates when strong nonlinear effects, including nonlinear refraction, mode competition and selffocusing/defocusing effects, may lead to laser instabilities and beam filamentation $^{25}$. Finding the optimized spatial profile of the pump beam that improves laser brightness, can be a guide in the design of patterned electrodes for electrical pumping ${ }^{26}$. Finally, such a controllable random laser itself offers a unique platform possessing many parameters with which to play (geometry, openness, local or non-uniform pumping, absorption, scattering ...) in the exploration 
of questions impossible or difficult to address with other lasers (for example, non-Hermitian systems, exceptional points in lasers ${ }^{27}$, coupled lasers, partially absorbing lasers, and so on).

\section{Methods}

Optofluidic random laser preparation. The PDMS optofluidic random lasers are fabricated following the soft lithography protocol described by Xia and Whitesides ${ }^{28}$. The geometric structure of the photomask shown in Fig. 1 is imprinted onto a $1 \mathrm{~mm}$-thick negative photoresist SU-8 mould. This mould is then used to replicate the polymer microstructures. A 10:1 PDMS:cross-linker mixture (Sylgard 184) is poured into the mould and then degassed for $10 \mathrm{~min}$ at a vacuum pressure of a few $\mathrm{mmHg}$, then cured at $90^{\circ} \mathrm{C}$ for $90 \mathrm{~min}$. Holes are perforated in the device to create inlets and the microchannel is bonded on a glass slide by plasma treatment.

Optofluidic random laser description. The gain medium is a $2.5 \times 10^{-3} \mathrm{M}$ ethanolic solution of Rhodamine $6 \mathrm{G}$ circulating in a $2.8 \mathrm{~mm}$-long PDMS microchannel comprised of a linear chain of randomly distributed rectangular PDMS pillars. The alternation of 70 layers, with two different indices of refraction ( $n_{\text {polymer }}=1.42$ and $n_{\text {dye }}=1.36$ ), provides multiple scattering and the necessary feedback to stimulate spontaneous emission into random laser emission. The microchannel is $80 \mu \mathrm{m}$ wide and $15 \mu \mathrm{m}$ deep. The pillars are $12 \mu \mathrm{m}$ thick and $40 \mu \mathrm{m}$ in length. Their random positions are uniformly chosen in the range $\pm 6 \mu \mathrm{m}$ around an arrangement having a period of $40 \mu \mathrm{m}$. In reality, the artificial spatial disorder we chose is unnecessary. The limited accuracy of the photolithographic process results in a $1 \mu \mathrm{m}$ tolerance in the position and thickness of the pillars, providing the necessary disorder at the optics scale, as shown in ref. 7. Because the random structure is static and the dye solution flows continuously within the microfluidic channel, dye-bleaching is reduced, making the lasing modes stable and the results reproducible over several hours. An auxiliary microchannel circulated by a separate dye flow $\left(5.0 \times 10^{-3} \mathrm{M}\right.$ ethanolic solution of Nile Blue) serves to image laser radiation scattered out by the structured channel (Fig. 1)

Experimental set-up and procedure. The laser beam from a frequency-doubled Q-switched Nd:YAG laser (Quantum Ultra: $532 \mathrm{~nm}, 10 \mathrm{~ns}$ pulse duration, maximum output energy $30 \mathrm{~mJ}$, repetition rate $10 \mathrm{~Hz}$ ) is expanded to uniformly illuminate the surface of the SLM (Holoeye LC 2002). The SLM itself, which sits between crossed polarizers to work in amplitude modulation, is placed in the object plane of a telescope with $5 \times$ reduction and is imaged on the sample after compression through a cylindrical lens with focal length $f=6 \mathrm{~mm}$. This set-up provides a $1.4 \mathrm{~mm}$-long, $4 \mu \mathrm{m}$-thick laser stripe line with nearly diffraction-free modulation down to $\mu \mathrm{m}$-large rectangular pixels. We chose to tightly focus the pump beam to a narrow line in order to enforce single transverse mode laser operation. The length of $1.4 \mathrm{~mm}$ has been chosen to limit the amplified spontaneous emission (ASE) and to provide a manageable modal density. We checked experimentally that doubling the length increases the ASE by a factor of three and the number of modes by a factor of two. The microchannel is precisely aligned with the laser stripe line under a Zeiss Axioexaminer microscope and imaged using a Hamamatsu Orca-R2 silicon CCD camera microscope. The laser emission is collected via an optical fibre connected to a Horiba iHR550 imaging spectrometer equipped with a $2,400 \mathrm{l} \mathrm{mm}^{-1}$ grating and a liquid nitrogen-cooled Symphony II camera (sampling rate $1 \mathrm{MHz}, 1,024 \times 56$ pixels, $26 \mu \mathrm{m}$ pixel pitch). The entrance slit is $50 \mu \mathrm{m}$. The resulting spectral resolution is $20 \mathrm{pm}$. The integrating time is $1 \mathrm{~s}$.

Optimization method. The image fed to the SLM is made up of 32 lines. Each line is grey-scale-coded on 256 levels and imaged onto the sample to form a $46 \mu \mathrm{m}$-long, $4 \mu \mathrm{m}$-thick pixel. We chose the 32 -column vectors $X_{i}$ of the $32 \times 32$ binary Hadamard matrix ${ }^{29,30}$ as the initial vertex to initiate the optimization procedure. Starting from any other basis on which an arbitrary 1D-pump profile, $f(x)$, can be decomposed should lead to convergence. We tested the alternate 'stripe basis' with vectors formed by contiguous on-pixels, all others being off. The algorithm also converges, but to a different solution. Our choice of the Hadamard basis is practical, as all elements (except the first one) have the same mean amplitude and therefore require a constant and reasonable pump fluence to reach threshold, provided we set a minimum uniform background illumination. The pump profile is therefore written as $f(x)=1 / 255\left[\alpha_{0}+\sum_{1 \rightarrow 32} \alpha_{i} X_{i}\right]$, where the $\alpha_{i}$ take discrete values in the range $[0,135]$ and the uniform background $\alpha_{0}=120$. Each vector $\left(\alpha_{i}\right)_{i \in[1,32]}$ corresponds to a particular pump profile associated with a particular emission spectrum $I(\lambda)$. To achieve single-mode operation at a desired wavelength, $\lambda$, we need to find the vector $\left(\alpha_{i}\right)_{i \in[1,32]}$ which maximizes the ratio $R\left(\lambda_{0}\right)=I\left(\lambda_{0}\right) / I\left(\lambda_{1}\right)$, where $\lambda_{1}$ corresponds to the wavelength of the lasing mode with highest intensity, apart from the mode at $\lambda_{0}$. Here, $I(\lambda)$ represents the intensity at $\lambda$ after the spectrum baseline has been subtracted, being defined as the intensity at $\lambda=555 \mathrm{~nm}$.
Experimentally, five spectra averaged over ten shots are acquired every second and then averaged for a given pump profile $f(x)$. The typical shot-to-shot variance of the lasing peaks is around $10 \%$. Thus averaging over 50 shots reduces spectrum fluctuations to roughly $1.4 \%$. Such accuracy is needed when the denominator in $R\left(\lambda_{0}\right)=\left(I\left(\lambda_{0}\right)\right) /\left(I\left(\lambda_{1}\right)\right)$ becomes small. Between successive acquisitions, the system is uniformly pumped $(f(x)=1)$ to 'clean up' the gain medium from any memory effect due to possible residual thermal effects from the previous pump profile. A typical optimization lasts $20 \mathrm{~min}$. After several hours ethanol infiltrates the PDMS and spectral shift becomes appreciable relative to the spectral precision of the measurement. This explains why different data sets were collected in Fig. 3b.

We checked the robustness of the solution and found that the optimization is lost $\left(R\left(\lambda_{0}\right)\right.$ divided by two) when the optimized pump profile is perturbed on every pixel by white noise with a standard deviation of $7 \%$. The algorithm, therefore, provides a sharp and accurate solution, sensitive to local perturbations.

Optimization algorithm. We use the Nelder-Mead simplex (direct search) method implemented in the fminsearch function of Matlab. We modified this function by setting the initial step (usual_delta parameter in fminsearch) to 0.5 to explore a large region of the 32-dimensional space. The number of pixels (32) has been determined for the best trade-off between sensitivity and computation time. It also has to be a power of two for definition of the Hadamard basis.

Received 2 January 2014; accepted 5 March 2014; published online 20 April 2014

\section{References}

1. Cao, H. Lasing in random media. Waves Random Media 13, R1-R39 (2003).

2. Wiersma, D. S. The physics and applications of random lasers. Nature Phys. 4, 359-367 (2008).

3. Wiersma, D. S. \& Noginov, M. A. Special issue on nano and random lasers. J. Opt. 12, 020201 (2010).

4. Wiersma, D. S. Disordered photonics. Nature Photon. 7, 188-196 (2013).

5. Bachelard, N., Andreasen, J., Gigan, S. \& Sebbah, P. Taming random lasers through active spatial control of the pump. Phys. Rev. Lett. 109, 033903 (2012).

6. Mosk, A., Lagendijk, A., Lerosey, G. \& Fink, M. Controlling waves in space and time for imaging and focusing in complex media. Nature Photon. 6, 283-292 (2012).

7. Bhaktha, S. B. N., Noblin, X., Bachelard, N. \& Sebbah, P. Optofluidic random laser. Appl. Phys. Lett. 101, 151101 (2012).

8. Redding, B., Choma, M. A. \& Cao, H. Speckle-free laser imaging using random laser illumination. Nature Photon. 6, 355-359 (2012).

9. Jin-Kyu Yang, J-K. et al. Lasing in localized modes of a slow light photonic crystal waveguide. Appl. Phys. Lett. 98, 241107 (2011).

10. Liu, B., Yamilov, A., Ling, Y., Xu, J. Y. \& Cao, H. Dynamic nonlinear effect on lasing in a random medium. Phys. Rev. Lett. 91, 063903 (2003).

11. Stano, P. \& Jacquod, P. Suppression of interactions in multimode random lasers in the Anderson localized regime. Nature Photon. 7, 66-68 (2013).

12. Van der Molen, K. L., Tjerkstra, R. W., Mosk, A. P. \& Lagendijk, A. Spatial extent of random laser modes. Phys. Rev. Lett. 98, 143901 (2007).

13. Türeci, H. E., Ge, L., Rotter, S. \& Stone, A. D. Strong interactions in multimode random lasers. Science 320, 643-646 (2008).

14. Andreasen, J., Sebbah, P. \& Vanneste, C. Nonlinear effects in random lasers. J. Opt. Soc. Am. B 28, 2947-2955 (2011).

15. Andreasen, J., Sebbah, P. \& Vanneste, C. Coherent instabilities in random lasers. Phys. Rev. A 84, 023826 (2011).

16. Kogelnik, H. \& Shank, C. V., Stimulated emission in a periodic structure. Appl. Phys. Lett. 18, 152-154 (1971).

17. Shank, C. V., Bjorkholm, J. E. \& Kogelnik, H. Tunable distributed-feedback dye laser. Appl. Phys. Lett. 18, 395-396 (1971).

18. Feng, J., Chen, T. R., Zhao, B. \& Yariv, A. Reduction of the frequency chirp of two section distributed feedback laser by nonuniform current injection. Appl. Phys. Lett. 66, 2028-2030 (1995).

19. Leonetti, M. \& López, C. Active subnanometer spectral control of a random laser. Appl. Phys. Lett. 102, 071105 (2013).

20. Vellekoop, I. M., Lagendijk, A. \& Mosk, A. P. Exploiting disorder for perfect focusing. Nature Photon. 4, 320-322 (2010).

21. Won, R. Optofluidics lasers: In random form. Nature Photon. 7, 3 (2013).

22. Andreasen, J., Vanneste, C., Ge, L. \& Cao, H. Effects of spatially nonuniform gain on lasing modes in weakly scattering random systems. Phys. Rev. A 81, 043818 (2010)

23. Andreasen, J. et al. Partially pumped random lasers. Int. J. Mod. Phys. B 28, 1430001 (2014)

24. Hisch, T, Liertzer, M., Pogany, D., Mintert, F. \& Rotter, S. Pump-controlled directional light emission from random lasers. Phys. Rev. Lett. 111, 023902 (2013). 
25. Ohtsubo, J. Semiconductor Lasers: Stability, Instability and Chaos 2nd edn (Springer-Verlag, 2008).

26. Liang, H. K. et al. Electrically pumped mid-infrared random lasers. Adv. Mater. 25, 6859-6863 (2013)

27. Liertzer, M. et al. Pump-induced exceptional points in lasers. Phys. Rev. Lett. 108, 173901 (2012).

28. Xia, Y. N. \& Whitesides, G. M. Soft lithography. Annu. Rev. Mater. Sci. 28, 153-184 (1998).

29. Sloane, N. J. A. \& Harwit, M. Masks for hadamard transform optics, and weighing designs. Appl. Opt. 15, 107-114 (1976).

30. Harwit, M. \& Sloane, N. J. A. Hadamard Transform Optics (Academic, 1979).

\section{Acknowledgements}

We thank J. P. Huignard, S. Bhaktha and J. Andreasen for useful discussions. We thank Y. Izmaylov-Mavrikova for her help in the sample microfabrication. P.S., N.B. and S.G. are grateful to the LABEX WIFI (Laboratory of Excellence within the French Program 'Investments for the Future') under reference ANR-10-IDEX-0001-02 PSL*. P.S. is grateful to the ANR under Grant No. ANR-08-BLAN-0302-01 and to the Groupement de Recherche 3219 MesoImage. S.G. is funded by the European Research Council (grant number 278025)

\section{Author contributions}

P.S. and S.G. initiated the study. X.N. designed and prepared the samples. N.B. set up the experiments and collected all the data in the laboratory of P.S. P.S. and N.B. analysed the data and prepared the manuscript. S.G. contributed to data interpretation and manuscript preparation.

\section{Additional information}

Supplementary information is available in the online version of the paper. Reprints and permissions information is available online at www.nature.com/reprints.

Correspondence and requests for materials should be addressed to P.S.

\section{Competing financial interests}

The authors declare no competing financial interests. 